\title{
Calibration of the Aerogel Tiles for the HELIX RICH
}

P. Allison ${ }^{1}$, J. J. Beatty ${ }^{1}$, L. Beaufore ${ }^{2}$, Y. Chen ${ }^{3}$, S. Coutu ${ }^{3}$, E. Ellingwood ${ }^{4}$, M. Gebhard ${ }^{5}$, N. Green ${ }^{6}$, D. Hanna ${ }^{4}$, B. Kunkler ${ }^{5}$, I. Mognet ${ }^{3}$, R. Mbarek ${ }^{2}$, K. McBride ${ }^{1}$, K. Michaels ${ }^{5}$, D. Müller ${ }^{2}$, J. Musser ${ }^{5}$, S. Nutter ${ }^{7}$, S. O’Brien ${ }^{4}$, N. Park ${ }^{8}$,

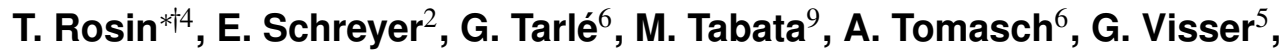
S. P. Wakely ${ }^{2}$, T. Werner ${ }^{2}$, I. Wisher ${ }^{2}$, and M. Yu ${ }^{3}$

${ }^{1}$ Ohio University, Columbus, OH 43210 USA

${ }^{2}$ University of Chicago, Chicago, IL 60637 USA

${ }^{3}$ Pennsylvania State University, University Park, PA 16802 USA

${ }^{4}$ McGill University, Montreal, Canada

${ }^{5}$ Indiana University, Bloomington, IN 47405 USA

${ }^{6}$ University of Michigan, Ann Arbor, MI 48109 USA

${ }^{7}$ Northern Kentucky University, Highland Heights, KY 41099 USA

${ }^{8}$ University of Wisconsin-Madison, Madison, WI 53706 USA

${ }^{9}$ Chiba University, Chiba, 263-8522, Japan

HELIX (High Energy Light Isotope eXperiment) is a balloon-borne experiment designed to measure the chemical and isotopic abundances of light cosmic ray nuclei, especially the ${ }^{10} \mathrm{Be} /{ }^{9} \mathrm{Be}$ ratio over the energy range from $0.2 \mathrm{GeV} / \mathrm{n}$ to beyond $3 \mathrm{GeV} / \mathrm{n}$. This is a key measurement for constraining cosmic-ray propagation models. The detector is a mass spectrometer, which is built around a 1 Tesla superconducting magnet and a high-resolution tracking system to determine particle rigidity. Time-of-flight counters and a ring-imaging Cherenkov detector (RICH) are used to measure velocities.

The proximity-focused RICH consists of a radiator made of aerogel tiles (refractive index approximately 1.15) and a detector plane of silicon photomultipliers. For discrimination of the ${ }^{9} \mathrm{Be}$ and ${ }^{10} \mathrm{Be}$ isotopes at high energy, the refractive index of the aerogel must be known to a precision of $0.07 \%$. Given the manufacturing tolerances in the production process, the index must be mapped over the lateral extent of aerogel tiles on a fine grid. In this contribution, we describe and show initial results from procedures developed for this task. These include laser-deflection and electron-beam measurements.

36th International Cosmic Ray Conference -ICRC2019-

July 24th - August 1st, 2019

Madison, WI, U.S.A.

\footnotetext{
*Speaker.

†thomas.rosin@mail.mcgill.ca
} 


\section{Introduction}

HELIX is a balloon-borne experiment designed to measure the chemical and isotopic abundances of light cosmic ray nuclei. Its main goal is to measure the ${ }^{10} \mathrm{Be} /{ }^{9} \mathrm{Be}$ ratio over the energy range from $0.2 \mathrm{GeV} / \mathrm{n}$ to beyond $3 \mathrm{GeV} / \mathrm{n}$. Given that ${ }^{10} \mathrm{Be}$ is radioactive (1.4 My half-life) while ${ }^{9} \mathrm{Be}$ is stable, but both are produced at the same time, in spallation of heavier nuclei like carbon on interstellar protons, the ratio can be used to constrain the propagation times of local cosmic rays. This observable can be used to help discriminate between different propagation models.

HELIX is scheduled for a long-duration ( $\sim 7$ days) stratospheric $(\sim 40 \mathrm{kmn}$ a.s.l.) balloon flight from McMurdo Station in Antarctica during the austral summer of 2020-21. The payload is a magnetic spectrometer based on a 1 Tesla superconducting magnet, formerly used in the HEAT experiment [1], within it a high-resolution drift-chamber tracking system is used to measure particle rigidity. A scintillator time-of-flight system is used to measure particle charge, and velocity at energies below $1 \mathrm{GeV} / \mathrm{n}$. At higher energies a RICH detector is used for velocity determination. More information about HELIX and some of its components can be found elsewhere in these proceedings.

\section{The HELIX RICH}

The RICH relies on a plane of aerogel tiles for the radiator and an array of silicon photomultipliers (SiPMs) for the detector plane [2]. The tiles are $100 \mathrm{~mm}$ by $100 \mathrm{~mm}$ in area and approximately $10 \mathrm{~mm}$ thick; a total of 36 will be used for the final flight. The aerogel is hydrophobic and has been designed to have a refractive index of approximately 1.15. It has been developed by M. Tabata [3] and is made using a novel pin drying technique [4].

In order to achieve $2.5 \%$ mass resolution at $3 \mathrm{GeV} / \mathrm{n}$, to resolve the beryllium isotopes sufficiently, we require a precise knowledge of the refractive index, at the level of $\Delta n / n \sim 7 \times 10^{-4}$. Manufacturing tolerances inherent in the aerogel production process result in variations of the index that are greater than this in a single tile so it is necessary to measure the index as a function of lateral position in each tile. We have decided to use two distinct methods to make such measurements and will use any discrepancies in results obtained as input to our estimates of systematic uncertainties. One method relies on measuring Cherenkov rings produced when a beam of electrons is sent through the tile and the other involves measuring the deflection of a laser beam as a function of the angle it makes with a tile. These will be described in the following sections.

Before being calibrated, the HELIX aerogel tiles are secured into aluminum frames, as shown in Figure 1, using a small amount of silicone adhesive (Dow Sylgard 184) at the edges. The frames, $1 \mathrm{~mm}$ thick and $12 \mathrm{~mm}$ deep provide hard edges for purposes of metrology and to protect the aerogel edges. Small tabs prevent the tile from coming loose once mounted in the payload, even if the adhesive degrades.

\section{Electron Beam Method}

The most direct method for measuring the refractive index of a tile is to place it in a highenergy particle beam and measure the radius of the resulting Cherenkov ring. This implies the 


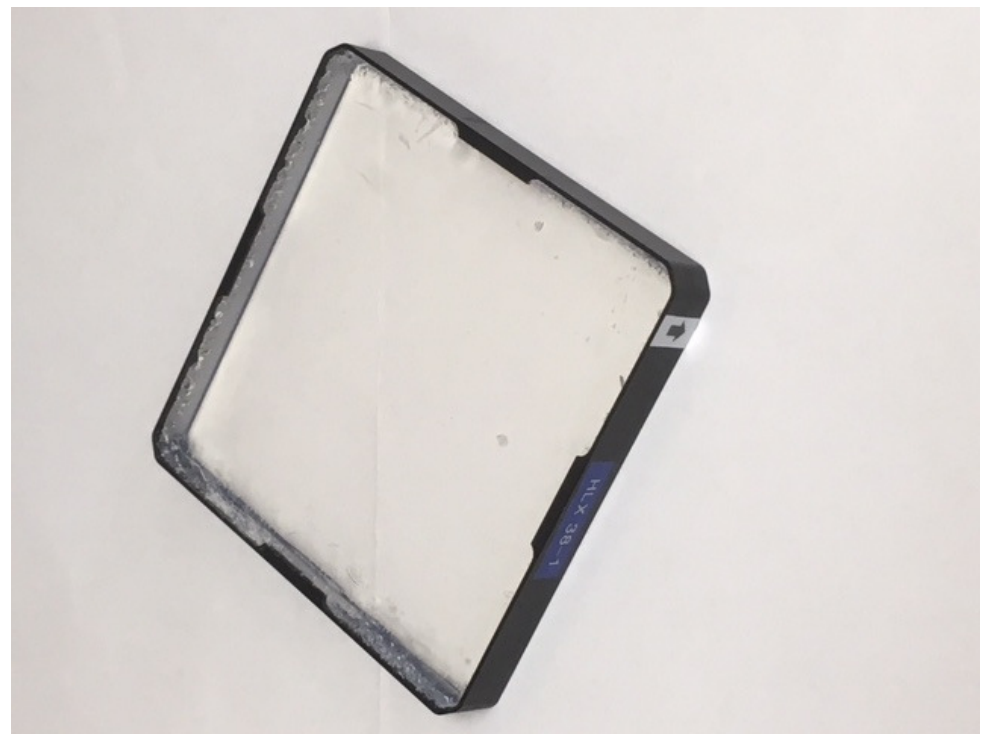

Figure 1: An aerogel tile mounted in its aluminum frame. The walls of the frame are $1 \mathrm{~mm}$ thick and the four visible tabs ensure that the tile cannot shake loose during transportation or flight.

existence of a detector plane to register the Cherenkov photons. The HELIX SiPM array is currently under fabrication and not available for aerogel calibration so we have constructed a detector plane using 16 one-dimensional CCDs from Toshiba (TCD-1304-DG) to sample the ring. These devices each have 3694 pixels and the pixels are $8 \mu \mathrm{m}$ long by $200 \mu \mathrm{m}$ wide. The active area is then 29.6 $\mathrm{mm}$ by $0.2 \mathrm{~mm}$. As shown schematically in Figure 2, the CCDs are arrayed in a circle with their mid-points at a radius of $200 \mathrm{~mm}$. Photons from the Cherenkov ring are sampled by the CCDs and used to fit the ring radius.

For the calibration beam we are using the Vickers $35 \mathrm{MeV}$ electron linac at the Ionizing Radiation Standards department of the National Research Council in Ottawa. The machine can deliver $2.5 \mu$ s pulses of electrons at rates upwards of 60 pulses per second. Pulses can have up to $12.5 \times 10^{9}$ electrons, so intensity is not a problem, even though most of the Cherenkov photons miss the CCDs. The beam is fully relativistic but undergoes more multiple scattering than higher energy (e.g., GeV) beams. According to our calculations this is not a limiting feature; beam width and divergence are more important and we are conducting collimation studies to improve these parameters.

For beam calibration, the aerogel tile is mounted on an X-Y scanning stage with $100 \mathrm{~mm}$ range in both coordinates. This allows beam-scan points over the entire surface of the tile. The CCD board is mounted downstream on an X-Y-Z scanning stage, with more limited ranges, to optimally position the Cherenkov ring on the CCDs.

NIM electronics, triggered by a timing signal from the linac control, are used to enable the CCD exposure for the duration of the beam pulse and to clock out the CCD pixel contents between pulses. The contents are digitized using 16 channels of Acqiris DC270 8-bit 1 GS/s digitizers. This data-acquisition system, as configured, can accommodate a rate of 80 pulses per second.

The calibration protocol foresees taking data on a grid of $19 \times 19$ points, spaced at $5 \mathrm{~mm}$ intervals over the face of the aerogel. At each point, sufficient statistics can be obtained with only 1 second of data and it is advisable to avoid irradiating a given point on the aerogel for too long in 


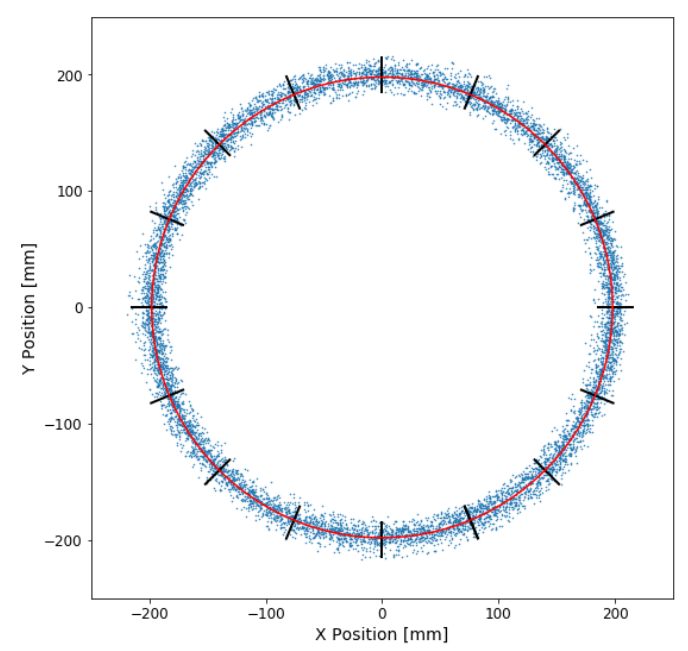

Figure 2: Schematic of the CCD-based device used to measure the Cherenkov ring generated by a calibration beam. The 16 black lines radiating from a common center represent the sensitive regions of one-dimensional CCDs and the blue dots show the impact points of Cherenkov photons coming from the upstream aerogel tile. The red circle is a fit to the coordinates of the photons hitting the CCDs.

case of radiation damage. Allowing for slewing to and settling at the next grid point and writing the data to disk, we can allocate a total of 15 seconds per point so an entire scan can be completed in an hour and a half.
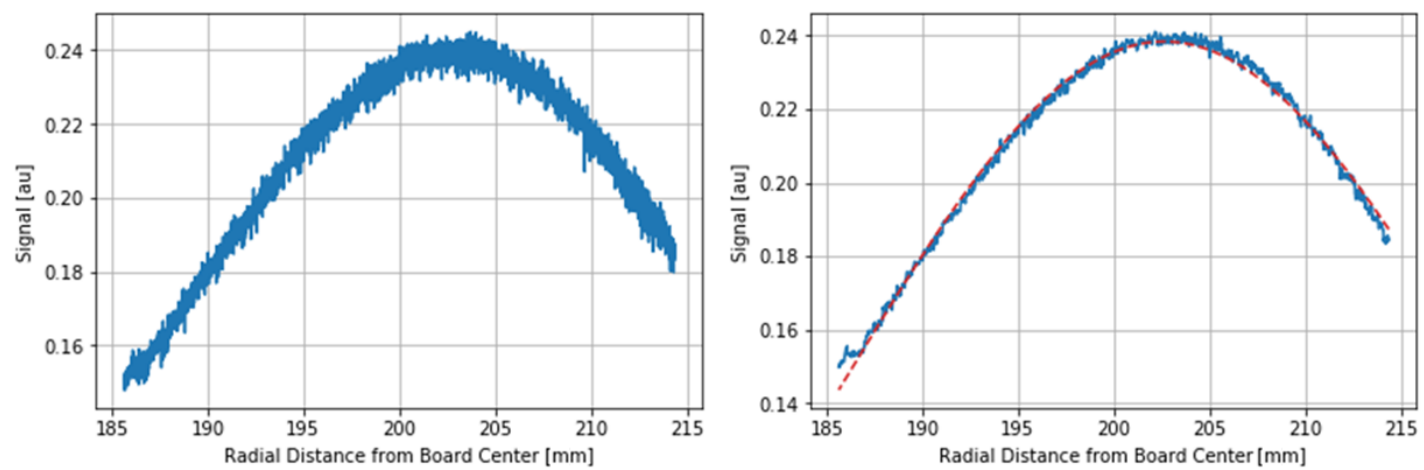

Figure 3: Left panel: Signal, in arbitrary units, vs radial distance from the board center in $\mathrm{mm}$, showing the whole readout frame for a single CCD, averaged over 100 beam pulses. The Cherenkov ring is broader than the length of the CCD but the region of maximum intensity is contained. Right panel: median values for groups of 10 pixels, to eliminate the non-statistical fluctuations seen in the left panel. The smooth line is a gaussian fit. 
A sample of data is shown in Figure 3 where the response of a single CCD to Cherenkov radiation from 100 pulses is shown in the left panel. Plotted vs radial distance from the board center in $\mathrm{mm}$ is the signal, in arbitrary units, in each pixel. Two features are evident; the distribution is very broad and is not contained within the CCD, and there are non-statistical fluctuations in the data. The first is due to beam divergence which will be corrected in future runs as we improve our tuning of the beam and introduce collimators. The origin of the fluctuations is not known but we can eliminate them by combining groups of 10 pixels and using the median of each group as an estimator of the light intensity in the region. This degrades the spatial resolution along the CCD, by a factor 10 , to $80 \mu \mathrm{m}$, still more than adequate for our purposes. The resulting distribution is shown in the right panel of Figure 3. It is well described by a gaussian which can be used to calculate the maximum of the distribution. The fit residuals can be used to calculate the uncertainty and this predicts a statistical uncertainty of $O\left(10^{-4}\right)$ in the measurement of the refractive index when data from 16 CCDs are combined.

\section{Laser Beam Deflection Method}

Another way to measure the refractive index of an aerogel tile is to measure the deflection of a laser beam as it traverses the tile at a finite incidence angle [5]. Referring to Figure 4, one can see that the deflection $d$ of a laser spot on a screen downstream from a tile of thickness $t$ depends on the angle $\theta_{i}$ between the incident direction of the laser beam and the normal to the tile's surface. Using Snell's Law, one can show that

$$
d\left(\theta_{i}, n\right)=t\left[\sin \left(\theta_{i}\right)-\cos \left(\theta_{i}\right) \tan \left(\sin ^{-1}\left(\frac{\sin \left(\theta_{i}\right)}{n}\right)\right)\right]
$$

To measure the refractive index we use the setup shown in Figure 4. The laser diode has a wavelength of $405 \mathrm{~nm}$ since we are interested in the UV-blue range where most of the Cherenkov spectrum lies. The CCD camera is a Prosilica 1380 with $1.4 \mathrm{M}$ pixels. The aerogel tile is mounted on a rotation stage, which itself is mounted on an X-Y scanning stage. For each X-Y point (we foresee a grid of points at $5 \mathrm{~mm}$ intervals) we acquire laser-spot images at five-degree intervals from $-50^{\circ}$ to $50^{\circ}$. Allowing $5 \mathrm{~s}$ for slewing and settling, a complete scan will take 11 hours.

The results of an angle scan at an arbitrary X-Y position are shown in Figure 5. It can be seen that the functional form described earlier is a good fit to the data.

A key measurement needed for the laser deflection method is the thickness of the aerogel tile as a function of lateral coordinate. This can be extracted from the fit to the deflection-vs-angle data but, given that there is considerable covariance with the refractive index value, we decided to map it separately. This is done using a Mitutoyo QV606 coordinate measuring machine located at the TRIUMF laboratory. This device is used to scan the tile on a $5 \mathrm{~mm}$ grid, measuring the height of the surface above a reference base. The tile is then flipped over and the procedure repeated. Results indicate a bowed structure with the middle of the surface being different from the edges by up to $0.5 \mathrm{~mm}$. A map of the thickness variation for a random tile is shown in Figure 6. The fact that the tile surfaces are not precisely parallel to each other means that small corrections will be needed to extract the exact value of the refractive index using this method. 


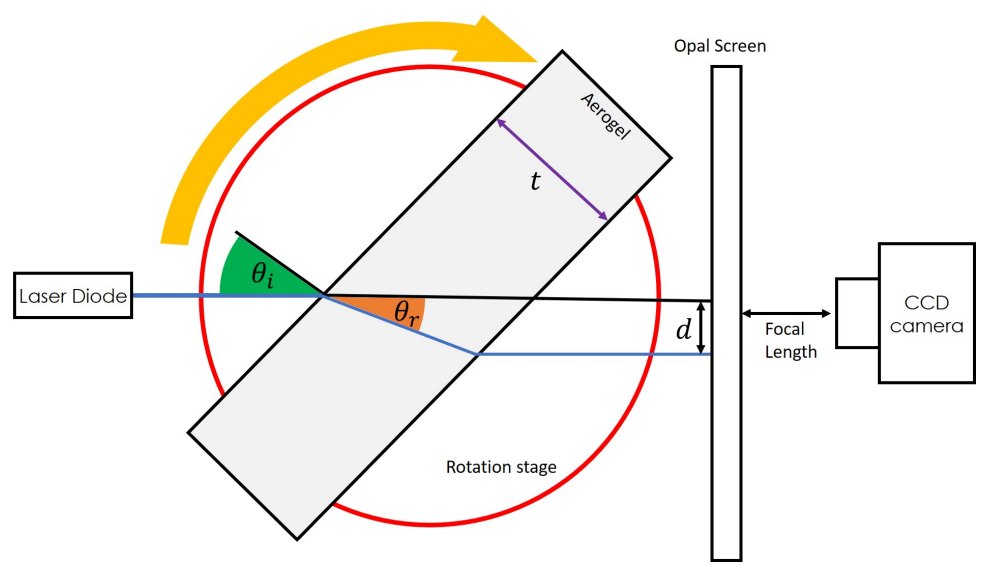

Figure 4: The setup used for measuring the refractive index using the deflection of a laser beam. The beam from a blue diode laser enters the tile at an angle $\theta_{i}$ and is is bent towards the normal, according to Snell's Law. It emerges from the tile on the other side and strikes an opal-glass diffusing screen. The image of the laser spot on the screen is recorded using a CCD camera.

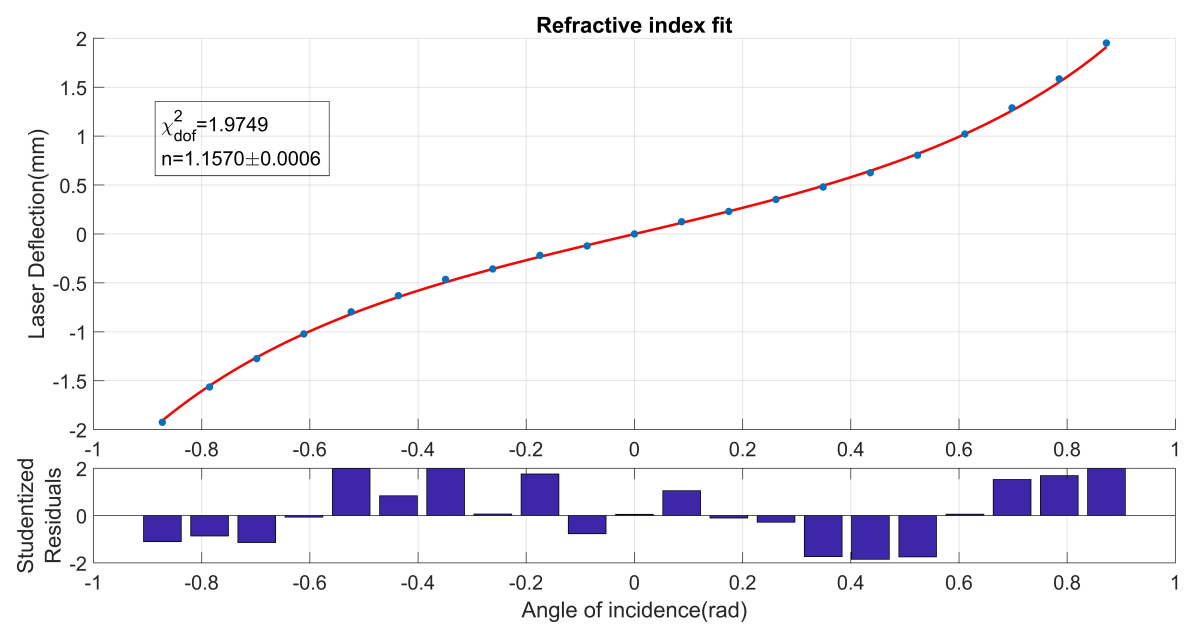

Figure 5: Laser point deflection as a function of tile angle for a single $\mathrm{X}-\mathrm{Y}$ scan position. Uncertainties are smaller than the symbols. The fitted function is that described in the text.

\section{Conclusions}

Mapping the refractive index of the aerogel tiles that will form the radiator plane of the HELIX RICH detector is an important task with a direct influence on the eventual quality of the primary measurement, the ${ }^{10} \mathrm{Be} /{ }^{9} \mathrm{Be}$ ratio. We have developed tools for undertaking this work and are ready to begin scans of tiles as they are delivered from our collaborator at Chiba University. Preliminary tests on prototype tiles indicate that the results will meet requirements and the time needed to carry out the calibrations is within the constraints of the project schedule. 


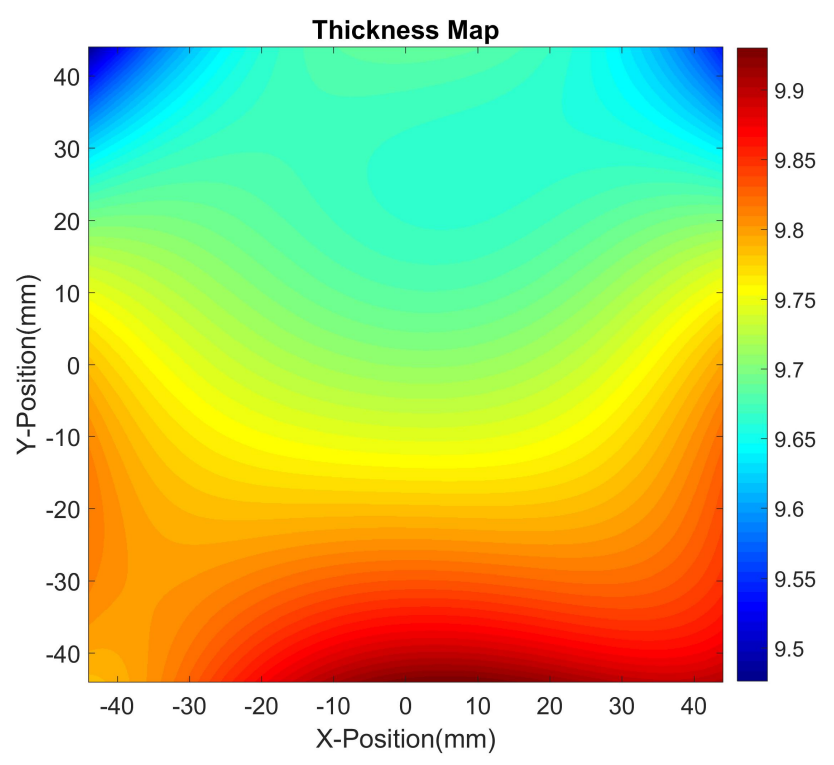

Figure 6: Thickness map for an arbitrary aerogel tile.

\section{Acknowledgements}

We thank M. McEwan and S. Walker at NRC for support during the electron beam studies and N. Hessey and T. Stack at TRIUMF for measuring the tiles with their CMM. HELIX research is supported by grants from NASA in the U.S. and by NSERC and the CSA in Canada.

\section{References}

[1] S. W. Barwick et al., The high-energy antimatter telescope (HEAT): An instrument for the study of cosmic ray positrons Nucl.Instrum.Meth. A400 (1997) 34-52

[2] I. Wisher for the HELIX Collaboration, Design and construction of the HELIX RICH detector, in 101 proceedings of the 36th International Cosmic Ray Conference, PoS(ICRC2019), to be published.

[3] M. Tabata et al., Developing a silica aerogel radiator for the HELIX ring-imaging Cherenkov system, arXiv:1901.06663

[4] M. Tabata et al., Development of Transparent Silica Aerogel Over a Wide Range of Densities, Nucl.Instrum.Meth. A 623(1) (2010) 339, arXiv:1112.5084

[5] D. Richter and D. Lipka, Measurement of the Refractive Index of Silica Aerogel in Vacuum, Nucl.Instrum.Meth. A 513(3) (2003) 635 